\title{
A New On-line Monitoring Method for Urban Refuse Based on Ultrasonic Principle
}

\author{
Jingxuan $\mathrm{Li}$ \\ School of Electrical and Electronic Engineering, North China Electric Power University, Beijing 102206, \\ China \\ ncepuljx@163.com
}

Keywords: Urban refuse, Ultrasonic principle, Large data analysis.

\begin{abstract}
Combined with the status quo and present problems of urban refuse, this paper proposes a new on-line monitoring method for urban refuse based on ultrasonic principle. Aiming at the low efficiency of urban refuse collection at present, we make a method to realize monitoring on-line by a low cost. This method use ultrasonic ranging system to monitor the amount of refuse in trash can and send the message to a monitoring center which can make a more effective plan for urban refuse collection. So this method can monitor urban refuse real-timely and improve the efficiency of collection.
\end{abstract}

\section{Introduction}

In recent years, the problem of urban refuse was getting worse day by day. Especially in big cities, the amount of refuse was increasing exponentially. The problems at present on urban refuse collection include collecting not in time, the low efficiency of collecting and so on. These problems, which are related to people's life and urban construction, seriously debase people's quality of life and the image of city. So we must pay attention to urban refuse and sovle the problems as quickly as possible.

This paper is based on the problems on urban refuse at present. It construct an on-line system based on ultrasonic principle to monitor urban refuse. The using of this system can get the condition of the trash cans real-timely and optimize the program of collection. So it can improve the efficiency of collecting and be useful to slove the present problems.

\section{The principle of the method}

The section headings are in boldface capital and lowercase letters. Second level headings are typed as part of the succeeding paragraph (like the subsection heading of this paragraph). All manuscripts must be in English, also the table and figure texts, otherwise we cannot publish your paper. Please keep a second copy of your manuscript in your office. When receiving the paper, we assume that the corresponding authors grant us the copyright to use the paper for the book or journal in question. When receiving the paper, we assume that the corresponding authors grant us the copyright to use the paper for the book or journal in question. When receiving the paper, we assume that the corresponding authors grant us the copyright to use.

This method use ultrasonic principle to realize the monitoring of trash can. Ultrasonic generator can shoot ultrasonic and it will reflect back when it encounter an obstacle. The reciver can get the ultrasonic reflected. 


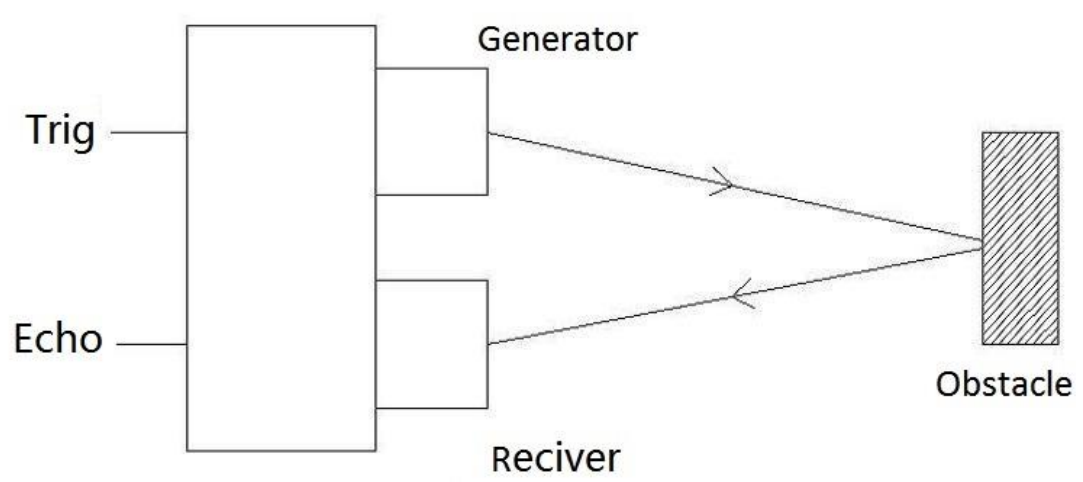

Fig. 1 Ultrasonic Distance Measurement Principle

If the time of shooting is $t \_1$, the time of reciving is $t \_2$, the speed of ultrasonic is $v$. When the ultrasonic generator and the reciver are in the same positon, we can calculate the distance between the generator and the obstacle with the three quantities:

$\mathrm{l}=0.5 \cdot \mathrm{v} \cdot\left(\mathrm{t}_{1}+\mathrm{t}_{2}\right)$.

If the ultrasonic generator and the reciver are at the top of the wall of the trash can and we make the ultrasonic shoot in the horizontal direction. If the trash can is not full, we can get the distance of the opposite wall, which is named 1_0; showen here;

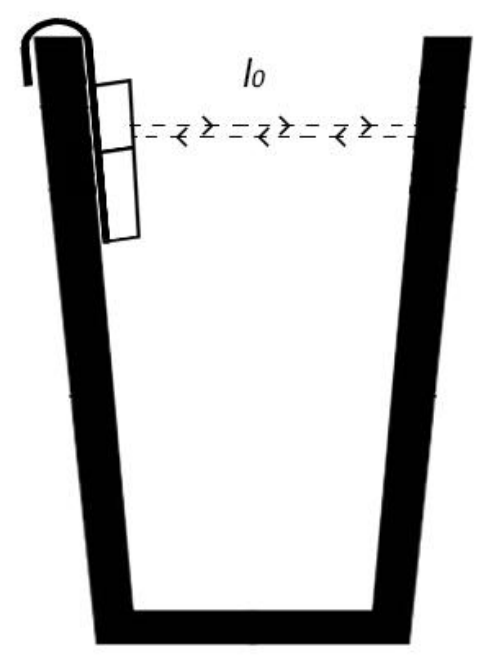

Fig.2 Measurement Principle used on a trash can

When the trash can is full, the distance we get is between the refuse and the wall, which is named l'. The relationship between 1_0 and l' is:

$l^{\prime}<1_{0}$.

If we set 0.510 as a threshold, we can judge the trash can is full or not by the distance we measure. So we realize the monitoring to trash can by the use of ultrasonic principle.

\section{The realization of the method}

The realization of this method not only need the theory, but also hardware design. For this problem, we design a device which can monitor the refuse on-line based on ultrasonic principle.

The design consists of two parts: 1. Refuse monitor, 2. On-line monitoring center.

Part 1: Refuse monitor is made of an ultrasonic distance measurement module, a MCS - 51 single chip computer, and two dry batteries and an EnOcean wireless module. The ultrasonic distance measurement module can measure the distance and sent it to the MCU, which is powered by the dry batteries. And the MCU sent the information to Part 2 by the wireless module. The schematic is as follows: 

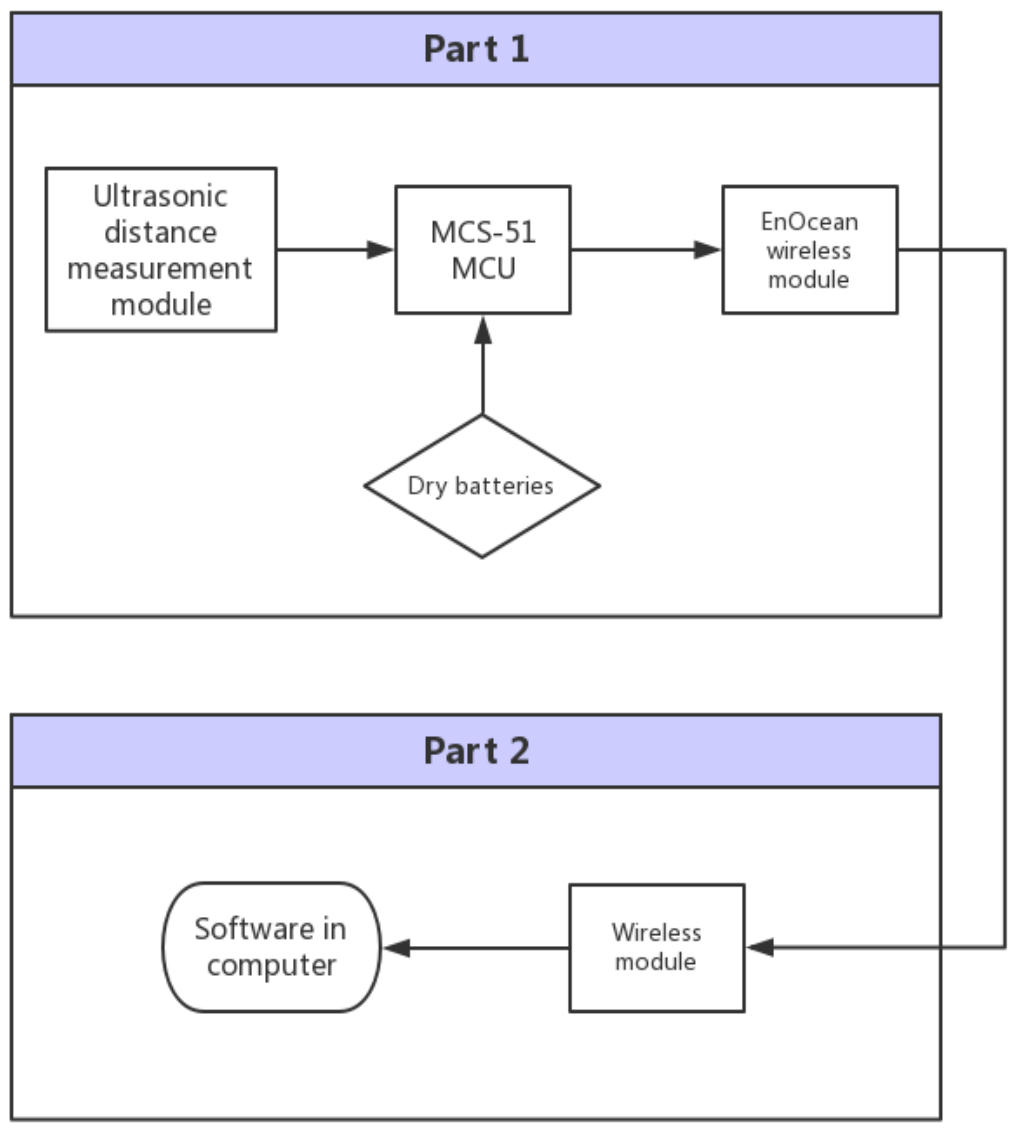

Fig. 3 Schematic

Part 2: On-line monitoring center is the software part. It use wireless module to recive the information from part 1 , then integrate it and send to computer. The monitoring center can show the condition of trash can on-line by software.

This design use part 1 to collect data and send it, and use part 2 to recive the data which is integrated by software. By this design, we can do monitoring the condition of trash cans within the area on-line.

Actually this method also have some weakness. It's transmission distance is limited, so the area can cover a whole city. And the refuse monitor part is susceptible to rain so it's waterproof ability should be improved. The power consumption of part 1 is still a little high, so we must replace the battery regularly.

\section{Summary}

In this paper, ultrasonic principle is fully used to measure distance. And we put forward a good design to realize the thory. This design is made of two parts, one is to collect information and the other one is to integrate data and realize the visible monitoring. With the help of this design, the efficiency of collection will be improved. And the problems of refuse collection at present will be better resolved.

Because of the weakness at present, there is room for improvement. First, we can expand the scope of communication by a cascade way. Second, we must improve it's waterproof ability so it can be used longer. Third, the MCU should stop running in a specific time to reduse it's power consumption. At last, this design should be used in a specific environment and find it's problems in using. Only by practice, this design can be popularized.

Urban refuse is a long-term problem which cannot be solved in a short time. The method put forward in this paper cannot sovle it right now, but it can advance the reform on the collection of urban refuse. 


\section{References}

[1]. Liu Ping,Tang Hongshou,Wang Rusong. Analysis ofTechnique and Economy for Urban Waste Incinerationin China. China Population, Resources and Environment. 2001

[2]. A.K.Gupta. Thermal Destruction of Solid Waste. Journal of Energy. 1996

[3]. Sirichai Watanasophon. Garbage Collection Robot on the Beach using Wireless Communications. Sarinee Ouitrakul. 2014

[4]. Hedeo Sugahara, Naoki Kamata,Masakazu Kato. The Optimal Plan for WTE Plants in an Urban Area Considering Refuse Collection and FIT. 